\title{
Decoding of spatiotemporal activity of auditory information in the cortex
}

\author{
Yusuke Hara', Yoshiki Kashimori ${ }^{1,2^{*}}$ \\ From Twentieth Annual Computational Neuroscience Meeting: CNS*2011 \\ Stockholm, Sweden. 23-28 July 2011
}

Animals utilize auditory information for survival and communications of conspecifics. A sequence of sound is analyzed in animals' brain as elementary components such as notes and syllables. It has been reported that auditory information is represented by spatiotemporal activity of primary auditory cortex [1]. However, how the elementary components of sound are encoded from the spatiotemporal activity of neurons is poorly understood. To address this issue, we present a model of auditory cortex, which performs a hierarchical processing of auditory information. The model consists of three layers of 2dimensional networks. We show that the aspects of the spatiotemporal activity in the primary cortex are encoded by a combination of feature-detective neurons and then by a dynamical attractor in higher-order cortex. The present study provides a clue for understanding the mechanism of how the information of notes and syllables are constructed from spatiotemporal activity of the primary auditory cortex.

We propose a neural network model for hierarchical processing of auditory information, which consists of three networks. The auditory information is encoded with spatiotemporal pattern of neuronal activity in the primary auditory (A1) cortex. The model of A1was made based on the previous model by Taniguch and Yamaguchi [2]. Then the spatiotemporal aspect of the neuronal activity is detected by the feature-detective (FD) neurons in the second layer. These FD neurons integrate the spatiotemporal pattern over a short time period, thereby enabling the second layer to represent the information about notes and about the correlation of notes. The information encoded by the FD neurons is then combined as a linkage of attractors in the feature binding

\footnotetext{
* Correspondence: kashi@pc.uec.ac.jp

${ }^{1}$ Graduate School of Information Systems, University of Electro-

Communications, Chofu, Tokyo 182-8585 Japan

Full list of author information is available at the end of the article
}

(FB) layer, providing a semantic information such as word.

\section{Results}

The model of the primary auditory cortex reproduced well the spatiotemporal activity of A1 neurons. The FD neurons integrated the spatiotemporal activity over a short time period and encoded the information about notes (i.e. "a","b", "c") and the correlation between the notes. The FB network combined the information about notes and their correlations as a linkage of dynamical attractors, enabling the network to represent the two words, "a-b-c" and "c-b-a". After the perception of 'a-b-c' or 'c-b-a', the network state recovered to a background state, in which the network state exhibited an itinerant state between the attractor "a-b-c" and "c-b-a".

\section{Conclusion}

We have shown a network model for hierarchical information processing in the auditory cortex, which consists of three layers. These layers encoded the information about different aspects of auditory features along the pathway from A1 to FB layer, enabling the system to percept a word in the FB layer. The present study provides an insight to understanding the information processing in auditory cortex.

\section{Author details \\ ${ }^{1}$ Graduate School of Information Systems, University of Electro- Communications, Chofu, Tokyo 182-8585 Japan. ${ }^{2}$ Dept. of Engineering Science, University of Electro-Communication, Chofu Tokyo 182-8585 Japan.}

Published: 18 July 2011

\section{References}

1. Taniguchi I, et al: Spatio-temporal pattern of frequency representation in the auditory cortex of guinea pigs. Neurosc. Lett 1992, 146:37-36.
C Biomed Central

(c) 2011 Hara and Kashimori; licensee BioMed Central Ltd. This is an open access article distributed under the terms of the Creative Commons Attribution License (http://creativecommons.org/licenses/by/2.0), which permits unrestricted use, distribution, and reproduction in any medium, provided the original work is properly cited. 
2. Yamagutchi $Y$, Taniguchi I: Neural activities as wave propagation in a neural network model of the auditory cortex. Tech. Rep. IEICE 1996, NC95141:197-204

doi:10.1186/1471-2202-12-S1-P139

Cite this article as: Hara and Kashimori: Decoding of spatiotemporal activity of auditory information in the cortex. BMC Neuroscience 201112 (Suppl 1):P139.

Submit your next manuscript to BioMed Central and take full advantage of:

- Convenient online submission

- Thorough peer review

- No space constraints or color figure charges

- Immediate publication on acceptance

- Inclusion in PubMed, CAS, Scopus and Google Scholar

- Research which is freely available for redistribution 